\title{
Blocking TRPA1 and TNF- $\alpha$ Signal Improves Bortezomib-Induced Neuropathic Pain
}

\author{
Chunrui Li Taoran Deng Zhen Shang Di Wang Yi Xiao \\ Department of Hematology, Tongji Hospital and Tongji Medical College, Huazhong University of \\ Science and Technology, Wuhan, China
}

\author{
Key Words \\ Chemotherapeutics $•$ Multiple myeloma $\cdot$ Bortezomib • Neuropathy $・$ TRPA1 $・$ TNF- $\alpha$
}

\begin{abstract}
Background/Aims: Bortezomib (BTZ) is largely used as a chemotherapeutic agent for the treatment of multiple myeloma. However, one of the significant limiting complications of BTZ is painful peripheral neuropathy during BTZ therapy. The purpose of this study was to examine the underlying mechanisms leading to neuropathic pain induced by BTZ. Methods: ELISA and western blot analysis were used to examine the levels of tumor necrosis factor alpha (TNF- $\alpha$ ) and its receptor, transient receptor potential ankyrin 1 (TRPA1) and intracellular p38MAPK and JNK signal in the lumbar dorsal root ganglion. Behavioral test was performed to determine mechanical pain and cold sensitivity in a rat model. Results: Systemic injection of BTZ significantly increased mechanical pain and cold sensitivity as compared with control animals $(P<0.05 \mathrm{vs}$. control rats). Our data also showed that protein expression of TRPA 1 was upregulated in the dorsal root ganglion of BTZ rats and blocking TRPA 1 attenuated mechanical pain and cold sensitivity in control rats and BTZ rats $(P<0.05$ vs. vehicle control). Notably, the inhibitory effect of blocking TRPA 1 on mechanical pain and cold sensitivity was smaller in BTZ rats than that in control rats. In addition, a blockade of TNF- $\alpha$ attenuated intracellular p38-MAPK and JNK signal in the dorsal root ganglion. This also decreased TRPA1 expression and alleviated mechanical hyperalgesia and cold hypersensitivity in BTZ rats. Conclusion: We revealed specific signaling pathways leading to neuropathic pain induced by chemotherapeutic BTZ. The data also suggest that blocking TRPA1 and tumor necrosis factor alpha is beneficial to alleviate neuropathic pain during BTZ intervention.

(C) 2018 The Author(s)

Published by S. Karger AG, Basel
\end{abstract}

\section{Introduction}

Bortezomib (BTZ), a reversible inhibitor of the proteasome complex, is used primarily as a chemotherapeutic agent for the treatment of multiple myeloma [1, 2]. However, clinical and animal studies have demonstrated that dose-limiting painful peripheral neuropathy is one of the significant adverse events observed during BTZ therapy [3]. In general, treatment options 
for these abnormal sensations have been restricted, partly due to a poor understanding of the underlying mechanisms responsible for neuropathic pain induced by chemotherapeutic agents such as BTZ. Drugs preventing and/or treating the painful symptoms evoked by BTZ therapy are lacking. Therefore, it is significant to determine molecular mediators of BTZ-induced painful neuropathy to make therapeutic strategies and have management of chemotherapeutic in patients with multiple myeloma.

Transient receptor potential ankyrin 1 (TRPA1) plays a functional role in regulating pain and neurogenic inflammation resulting from channel activation to a variety of compounds including pungent agents, irritant chemicals, reactive oxygen, and products of oxidative stress-induced lipid peroxidation [4-8]. TRPA1 has been shown to appear in dorsal root ganglion (DRG) neurons [7] and is engaged in development of mechanical hypersensitivity and painfully cold temperature $[9,10]$. TRPA 1 has also been reported to mediate mechanical hyperalgesia and cold hypersensitivity induced by chemotherapeutic agents [11]. Thus, in this report, we postulated that sensory TRPA1 plays a role in regulating mechanical and cold sensitivity in rats that received BTZ. We hypothesized that injection of BTZ amplifies protein expression of TRPA1 receptor in the DRG thereby resulting in mechanical hyperalgesia and cold hypersensitivity. Blocking TRPA1 attenuates mechanical hyperalgesia and cold hypersensitivity observed in BTZ rats.

Pro-inflammatory cytokines, such as tumor necrosis factor alpha (TNF- $\alpha$ ) play an important role in facilitating the development of neuropathic pain $[12,13]$. In numerous neuropathic pain models, TNF- $\alpha$ expression in sensory neurons appears to be upregulated following peripheral nerve injury [14]. Local application of TNF- $\alpha$ induces persistent hyperalgesia and allodynia in naive rats [15]. The application of chemotherapeutic drugs including such as paclitaxel or vincristine also amplifies the expression of TNF- $\alpha$ [15]. Inhibition of TNF- $\alpha$ by inhibitor or genetic impairment attenuates mechanical hyperalgesia and allodynia [14]. Moreover, recent studies have shown that BTZ treatment increases TNF- $\alpha$ expression in DRG and spinal dorsal horn $[16,17]$ and antibody against TNF- $\alpha$ reduces BTZ-induced allodynia in rats [18]. However, it is unknown whether TNF- $\alpha$ is engaged in BTZ-induced peripheral neuropathy via TRPA1 signaling. Thus, in this report, we examined the effects of TNF- $\alpha$ inhibition on protein expression of TRPA1 in DRG and on mechanical and cold sensitivity in BTZ rats. We hypothesized that inhibition of TNF- $\alpha$ attenuates intracellular p38-MAPK and JNK signal in DRG and thereby decreases the protein expression levels of TRPA1 and further alleviates BTZ-induced mechanical hyperalgesia and cold hypersensitivity.

\section{Materials and Methods}

\section{Ethic statement}

All animal protocols were in accordance with the guidelines of the International Association for the Study of Pain and approved by the Research Committee of our institution. In order to minimize experimental bias due to the sex difference, both genders of Wistar rats (200-250 g; 50\% male vs. 50\% female) were housed in individual cages with free access to food and water. The animals were kept in a temperaturecontrolled room $\left(25^{\circ} \mathrm{C}\right)$ on a $12 / 12 \mathrm{~h}$ light/dark cycle.

\section{A model of neuropathic pain and administration of drugs}

BTZ $(0.4 \mathrm{mg} / \mathrm{kg}$ body weight; Haoran BioTech Co., Shanghai, China; Cat\#B1408) was administrated intraperitoneally (i. p.) once daily for 5 consecutive days on the basis of previous reports [16, 19]. Control animals received an equivalent volume of saline. After BTZ intervention, TRPA1 antagonist HC030031 (1, 3, 10 mg/kg body weight; Sigma, St. Louis, MO, US; Product\#H4415/CAS\#349085-38-7) was administrated i.p. each day for the following 3 consecutive days. In the same fashion, a TNF- $\alpha$ synthesis inhibitor, pentoxifylline (PTX, 10, 20 and 40 mg/kg body weight; Sigma, St. Louis, MO, US; Product\# P1784/CAS\#6493-05-06) was given i.p. each day for 3 consecutive days.

Behavioral test

To quantify the mechanical sensitivity of the hindpaw, rats were placed in individual plastic boxes and allowed to acclimate for $>30 \mathrm{~min}$. Mechanical paw withdrawal threshold (PWT) of rat hindpaw in response 
to the stimulation of von Frey filaments was determined. A series of calibrated von Frey filaments (ranging from 0.4 to 18 g; BioSeb Co., Vitrolles, France; model Bio-VF-M) were applied perpendicularly to the plantar surface of the hindpaw with an appropriate force to bend the filaments until paw withdrew. Note that the filaments were bent for 5-10 s in our protocols and in general they can be bent sufficiently for $\sim 60 \mathrm{~s}$. In the presence of a response, the filament of next lower force was applied. In the absence of a response, the filament of next greater force was applied. To avoid injury during tests, the cutoff strength of the von Frey filament was $18 \mathrm{~g}$. The tactile stimulus producing a 50\% likelihood of withdrawal was determined using the "up-down" method [20]. Each trial was repeated 2 times at approximately 2 min intervals. The mean value was used as the force produced a withdrawal response.

To examine cold sensitivity, Thermal Place Preference System (Coulburn Instruments) was used to perform the thermal place preference test in order to assess a cold avoidance behavior. Two connecting metal plates were surrounded by a plastic enclosure. The first plate was kept at neutral temperature $\left(25^{\circ} \mathrm{C}\right)$ and the second plate was kept at cold temperature $\left(12^{\circ} \mathrm{C}\right)$. The test was performed in darkness and each session lasted 3 minutes. During the session, the rats were left free to explore both plates. The time spent on the cold plate during the entire session was recorded using an infrared camera connected to a computer to determine cold avoidance behavior. Thus, cold sensitivity was expressed as percentage time spent on the cold plate over 3 minutes [time on cold plate (seconds)/180 seconds $\mathrm{x} \%$ ]. To better control behavior test, the rats were repeatedly placed on the apparatus with both plates held at room temperature $\left(25^{\circ} \mathrm{C}\right)$ during 3 minutes 2 days before the beginning of the experiment. Note that rats spent an equal amount of time on each plate under these conditions, suggesting that animals showed no place preference. Also, to avoid learning or any place preference unrelated to cold, the temperature of the plates were inverted between two consecutive sessions. Two trials were performed for each of dosages and data were averaged. It is noted that the animal experiments were conducted blindly in this report.

\section{ELISA measurements}

All the tissues from individual rats were sampled for the analysis. In brief, DRG tissues (L4-L6) of the rats were removed. Total protein was then extracted by homogenizing the sample in ice-cold immunoprecipitation assay buffer with protease inhibitor cocktail kit (Promega Co. Madison, WI; Cat\# G6521). The lysates were centrifuged at 15, $000 \mathrm{xg}$ for $15 \mathrm{~min}$ and the supernatants were collected for measurements of protein concentrations using a bicinchoninic acid assay reagent kit.

The levels of TNF- $\alpha$ were examined using an ELISA assay kit (Wuhan Fine Biotech Co., Cat\# ER1393) according to the provided description and modification. Briefly, polystyrene 96 -well microtitel immunoplates were coated with affinity-purified rabbit anti-TNF- $\alpha$ antibodies. Parallel wells were coated with purified rabbit IgG for evaluation of nonspecific signal. After overnight incubation, plates were washed. Then, the diluted samples and TNF- $\alpha$ standard solutions were distributed in each plate. The plates were washed and incubated with anti-TNF- $\alpha$ galactosidase. Then, the plates were washed and incubated with substrate solution. After incubation, the optical density was measured using an ELISA reader.

\section{Western blot analysis}

Briefly, DRG tissues (L4-L6) were removed and total protein was extracted. The lysates were centrifuged at $15,000 \mathrm{xg}$ for $15 \mathrm{~min}$ and the supernatants were collected. After being denatured, the supernatant samples containing $20 \mu \mathrm{g}$ of protein were loaded onto gels and electrically transferred to a polyvinylidene fluoride membrane. The membrane was incubated overnight with primary antibodies: rabbit anti-TNFR1 (1:500, Abcam \#ab90463), anti-TRPA1 (1:500, Novus Bio, NB100-91319), anti-p-p38-MAPK (1:500, USBio, USB\#403230)/ p38-MAPK (1:500, USBio, USB\#403226) and anti-p-JNK1(1:500, Abcam \#ab47337)/ JNK1 (1:500, Abcam \#ab213521). The membranes were washed and incubated (8 hours) with an alkaline phosphatase conjugated anti-rabbit secondary antibody (1:1000, Sigma, Cat\#A3687). The immunoreactive proteins were detected by enhanced chemiluminescence. The bands recognized by the primary antibody were visualized by exposure of the membrane onto an x-ray film. The membrane was stripped and incubated with anti- $\beta$-actin to show equal loading of the protein. The film was then scanned and the optical density of TRPA1/TNFR1/p-p38-MAPK/ p38-MAPK/p-JNK1/JNK1/ $\beta$-actin bands was analyzed using the Scion Image software.

\section{Statistical analysis}

All data were analyzed using a two-way repeated-measures analysis of variance. Values were presented as means \pm standard error of mean. As appropriate, Tukey's post hoc tests were used. For all analyses, differences were considered significant at $P<0.05$. All statistical analyses were performed by using SPSS for Windows version 13.0 (SPSS Inc.). 


\section{Results}

\section{Basal levels of paw withdrawal threshold (PWT)}

Fig. 1 is a schematic diagram illustrating the timetable giving drugs and experimental protocols in the study. Table 1 demonstrates basal levels of PWT for 5 consecutive days after BTZ was injected in rats. No significant differences in PWT were observed between male and female. We used both sex of animals in all experiments of this report. BTZ injection significantly decreased PWT as compared with saline control. i.e., PWT was $8.7 \pm 0.5 \mathrm{~g}$ in control rats and $4.6 \pm 0.5 \mathrm{~g}$ in BTZ rats $(P<0.05 \mathrm{vs}$. control rats $)$ on the day when antagonists, HC030031 and PTX were given. This result suggests that pain was developed in rats after administration of BTZ.

\section{Effects of blocking TRPA1 on mechanical and cold sensitivity}

The effects of BTZ on PWT were analyzed using two-way repeated-measures analysis of variance. Fig. 2 shows that PWT appeared to be smaller in BTZ rats than that in control rats for saline and three dosages of HC030031 $(1,3,10 \mathrm{mg} / \mathrm{kg})$ at different time courses (0-7 days after HC030031). This figure also demonstrates that PWT was increased in a dosedependent way after injection of HC030031 (1, 3, $10 \mathrm{mg} / \mathrm{kg})$ in control rats and $\mathrm{BTZ}$ rats. As 10 $\mathrm{mg} / \mathrm{kg}$ of HC030031 was given, the effects were observed 1 day after its administration, and peaked at day 3 after injection. The effects began to reduce after injection was discontinued. In addition, the percentage increase of PWT evoked by HC030031 was smaller in BTZ rats than that in control rats. i.e. as $10 \mathrm{mg} / \mathrm{kg}$ of HC030031 was given, PWT was increased by $46 \%$ in BTZ rats $(P<0.05$, control rats $/ \mathrm{n}=12$ vs. $\mathrm{BTZ}$ rats $/ \mathrm{n}=15$ ) and $79 \%$ in control rats 3 days after the first injection of HC030031.

In addition, Fig. 2 shows that BTZ injection significantly decreased $\%$ time spent on the cold plate as compared with saline injection. Increases in cold sensitivity were greater in BTZ rats than these increases in control rats for saline and three dosages of HC030031 The percentage time spend was $45 \pm 6 \%$ in control rats and $30 \pm 5 \%$ in BTZ rats on the day before HC030031 was given $(P<0.05 v s$. controls). This figure further shows that blocking TRPA1

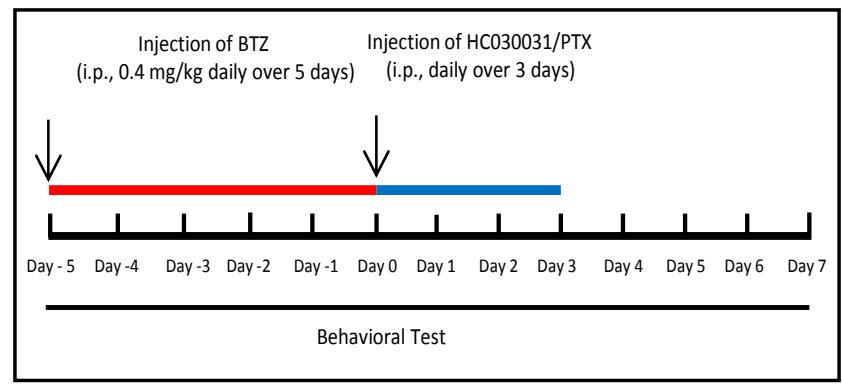

Fig. 1. A schematic diagram showing the schedule giving drugs and experimental protocols. BTZ wasinjected [intraperitoneally (i.p.), daily, $0.4 \mathrm{mg} / \mathrm{kg}$ body weight] over 5 days (indicated by red bar). The starting day is expressed as "day -5 ". An equivalent volume of saline was given in control animals. Then, HC030031 and PTX were administered (indicated as blue bar) at the end of BTZ injection (day 0), which was also marked as day 0 as indicated in Fig. 2. and Fig. 3.

Table 1. Basal paw withdrawal threshold (PWT) in control rats and rats during BTZ intervention prior to administration of HC030031 and PTX. * $\mathrm{P}<0.05$ vs. day -0 ; and $\dagger \mathrm{P}<0.05$ vs. saline control for each day. The total number of control rats $=78$ (39 for each of male and female); and the total number of BTZ rats $=87$ ( 44 for male and 43 for female). No significant differences were observed between male rats and female rats for control and BTZ injection. The effects of BTZ on PWT were analyzed using two-way repeated-measures analysis of variance. Note that administration of HC030031 and PTX was started on day 0 (this is also indicated in Fig. 2 and Fig. 3)

\begin{tabular}{lcccccc}
\hline PTW (g) & Day -5 & Day -4 & Day -3 & Day -2 & Day -1 & Day 0 \\
\hline Control & & & & & & \\
Male & $8.6 \pm 0.7$ & $8.9 \pm 0.8$ & $8.8 \pm 0.7$ & $9.0 \pm 0.9$ & $9.2 \pm 0.7$ & $8.9 \pm 0.8$ \\
Female & $8.4 \pm 0.8$ & $8.5 \pm 0.7$ & $8.4 \pm 0.6$ & $8.6 \pm 0.8$ & $8.6 \pm 0.6$ & $8.5 \pm 0.5$ \\
BTZ & & & & & & \\
Male & $8.7 \pm 0.9$ & $7.8 \pm 0.9$ & $6.6 \pm 0.5^{*}+$ & $5.3 \pm 0.6^{*}+$ & $4.5 \pm 0.8^{*} \dagger$ & $4.7 \pm 0.7^{*} \dagger$ \\
Female & $8.5 \pm 0.8$ & $7.4 \pm 0.7$ & $6.4 \pm 0.6^{*}+$ & $5.1 \pm 0.7^{*} \dagger$ & $4.5 \pm 0.7^{*}+$ & $4.5 \pm 0.6^{*} \dagger$ \\
\hline
\end{tabular}




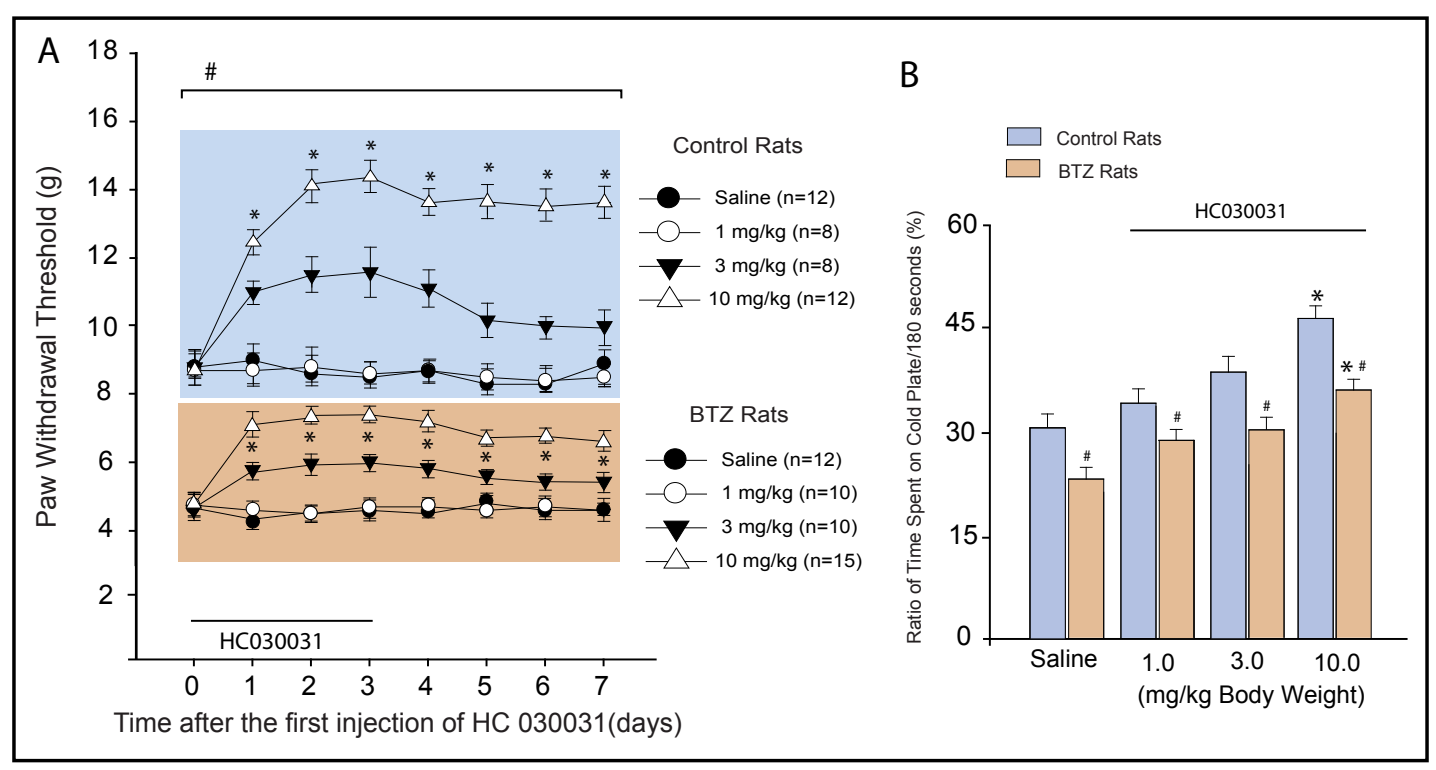

Fig. 2. Effects of blocking TRPA1 on mechanical and cold sensitivity. TRPA1 was blocked by administration of HC030031 (1, 3, $10 \mathrm{mg} / \mathrm{kg}$ body weight; i.p. each day over 3 consecutive days). Paw withdrawal threshold (PWT, g) and cold sensitivity expressed as time spent on the cold plate (\%) were examined in control rats and BTZ rats. (A): PWT was smaller in BTZ rats (presented in brown box) than that in control rats (presented in grey box) for saline and three dosages of HC030031 at different time courses. As compared with saline injection, HC030031 increased PWT in control rats and BTZ rats. Note that increases of PWT evoked by attenuation of TRPA 1 appear to be less degree in BTZ rats than these in control rats. ${ }^{*} \mathrm{P}<0.05$ vs. saline control and other dosages. \# $\mathrm{P}<0.05$, BTZ rats with injection of saline and three dosages of HC030031 vs. respective control rats with injection of saline and three dosages of HC030031 over 7 days after start of HC030031 (two-way repeated-measures analysis of variance was used for statistical analysis). The number of rats in each group was shown on the figure. (B): showing that time spent on the cold plate was less in BTZ rats than in control rats for saline and three dosages of HC030031. HC030031 elevated \% time spent on the cold plates in control rats and BTZ rats 3 days after its injection as compared with saline injection. The effects of HC030031 on cold sensitivity appeared to be less degree in BTZ rats than these in control rats. ${ }^{*} \mathrm{P}<0.05$ vs. saline control and other dosages. $\# \mathrm{P}<0.05$, BTZ rats vs. control rats for saline and three dosages of HC030031 three days after injection of HC030031.

with $\mathrm{HC} 030031(10 \mathrm{mg} / \mathrm{kg})$ from day 0 to day 3 significantly attenuated cold sensitivity to a greater degree in control rats (\% time increased by $81 \%, \mathrm{n}=12$ ) than in BTZ rats $(\%$ time increased by $48 \%, \mathrm{n}=15, P<0.05 \mathrm{vs}$. control rats).

\section{Effects of blocking TNF- $\alpha$ on mechanical and cold sensitivity}

Two-way repeated-measures analysis of variance was employed to analyze the effects of BTZ on PWT. Fig. 3 shows that PWT appeared to be smaller in BTZ rats than that in control rats for saline and three dosages of PTX $(10,20,40 \mathrm{mg} / \mathrm{kg})$ at different time courses. We further examined the role played by inhibition of TNF- $\alpha$ in attenuating mechanical and cold sensitivity. This figure further shows that injection of PTX attenuated mechanical sensitivity in BTZ rats and control rats as compared with saline injection. The inhibitory effects of PTX $(10,20,40 \mathrm{mg} / \mathrm{kg})$ on mechanical sensitivity appeared in a dose-dependent way. When 40 $\mathrm{mg} / \mathrm{kg}$ of PTX was injected, the effects were observed 1 day after its administration and peaked at day 3 The effects began to reduce after injection was discontinued. The effects were smaller in BTZ rats than in control rats $(P<0.05$, BTZ vs. controls). In addition, Fig. 3 shows that cold sensitivity was increased in BTZ rats as compared with control rats for saline and three dosages of PTX. Inhibition of TNF- $\alpha$ with PTX $(40 \mathrm{mg} / \mathrm{kg})$ attenuated cold sensitivity to a greater degree in control rats than in BTZ rats $(P<0.05, \mathrm{BTZ}$ vs. controls; $\mathrm{n}=12$ in each group). 


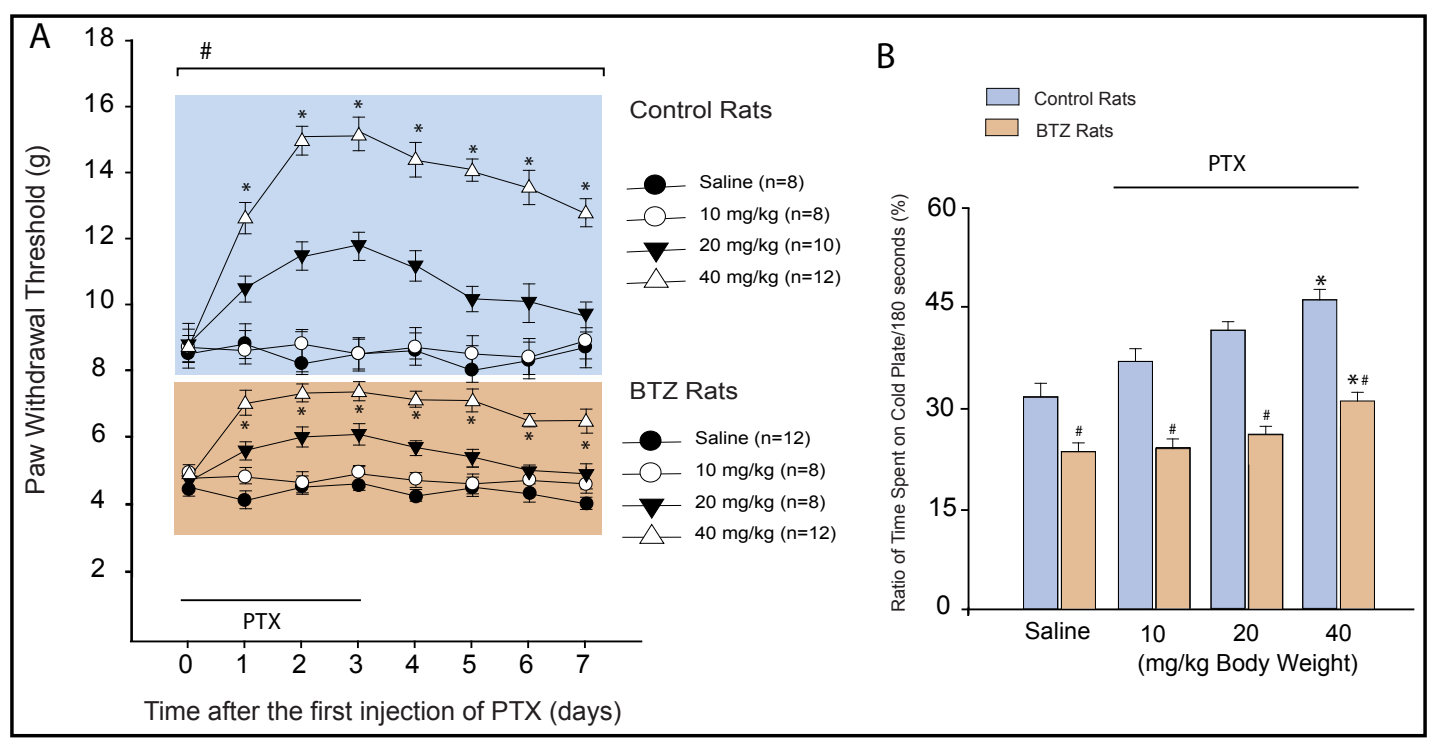

Fig. 3. Effects of blocking TNF- $\alpha$ on mechanical and cold sensitivity. TNF- $\alpha$ was inhibited by pentoxifylline (PTX; 10, 20, $40 \mathrm{mg} / \mathrm{kg}$ body weight; i.p. each day over 3 consecutive days). Paw withdrawal threshold (PWT) and cold sensitivity expressed as time spent on the cold plate (\%) were examined in control rats and BTZ rats. (A): PWT was smaller in BTZ rats (indicated in brown box) than in control rats (indicated in grey box) for saline and three dosages of HC030031 at different time courses. PTX increased PWT in control rats and BTZ rats as compared with saline control, but the amplitude of PWT increases evoked by PTX was smaller in $\mathrm{BTZ}$ rats than that in control rats. ${ }^{*} \mathrm{P}<0.05$ vs. saline and other dosages. \# $\mathrm{P}<0.05$, $\mathrm{BTZ}$ rats with injection of saline and three dosages of PTX vs. respective control rats with saline and three dosages of PTX over 7 days after start of PTX (two-way repeated-measures analysis of variance was used for statistical analysis). The number of rats in each group was shown on the figure. (B): showing that time spent on the cold plate was less in BTZ rats than in control rats for saline and three dosages of PTX. PTX elevated \% time spent on the cold plates in control rats and BTZ rats 3 days after its injection. The effects of PTX on cold sensitivity appeared to be less degree in BTZ rats than these in control rats. ${ }^{*} \mathrm{P}<0.05$ vs. saline and other dosages. \# P<0.05, BTZ rats vs. control rats for saline and three dosages of PTX 3 days after injection of PTX.

\section{Effects of blocking TNF- $\alpha$ on TRPA1 signal leading to neuropathic pain}

The effects of TNF- $\alpha$ on TRPA1 signal pathway were examined in additional groups. In this experiment, DRG tissues were removed three days after the beginning injection of PTX. First, the levels of TNF- $\alpha$ and expression of TNFR1 and TRPA1 were examined in the DRG of control rats with injection of PTX. PTX decreased TNF- $\alpha$, TNFR1 and TRPA1 in control animals. i.e., TNF- $\alpha$ was $115 \pm 15 \mathrm{pg} / \mathrm{mg}$ protein in the DRG of control rats $(\mathrm{n}=5)$; and $75 \pm 9$ $\mathrm{pg} / \mathrm{mg}$ protein in the DRG of control rats with PTX $(\mathrm{n}=6 ; P<0.05$ vs. control rats without PTX). Likewise, expression of TNFR1 and TRPA1 normalized to $\beta$-actin was $1.03 \pm 0.21$ and $1.06 \pm 0.18$ in the DRG of control rats; and $0.68 \pm 0.12$ and $0.72 \pm 0.16$ in the DRG of control rats with PTX $(P<0.05$ vs. control rats without PTX; $n=6-8$ in each group).

Moreover, Fig. 4 demonstrates that BTZ administration amplified the levels of TNF- $\alpha$ in DRG as compared with control rats $(P<0.05$, BTZ rats vs. control rats, $n=10$ in control; and $n=12$ in BTZ group). BTZ also increased protein expression of TNFR1 (a subtype TNF- $\alpha$ receptor) and TRPA1 as compared with control rats $(P<0.05$, BTZ rats $v s$. control rats, $\mathrm{n}=6$ 8 in each group). As PTX was given, the levels of TNF- $\alpha$ were decreased, which likely led to a less stimulation on TRFR1 and reduction of its expression. Fig. 4 further shows that PTX attenuated upregulation of TNFR1 and TRPA1 induced by BTZ $(P<0.05 v s$. BTZ without PTX). Moreover, intracellular signal pathways of DRG neurons, namely, p38-MAPK and JNK, were examined. BTZ upregulated phosphorylated p38-MAPK and JNK and these amplifications were inhibited by administration of PTX. It is noted that total protein levels of p38-MAPK and JNK were not increased significantly by BTZ, but the ratio of phosphorylated p38-MAPK and JNK and total phosphorylated p38-MAPK and JNK was significantly increased by BTZ. 


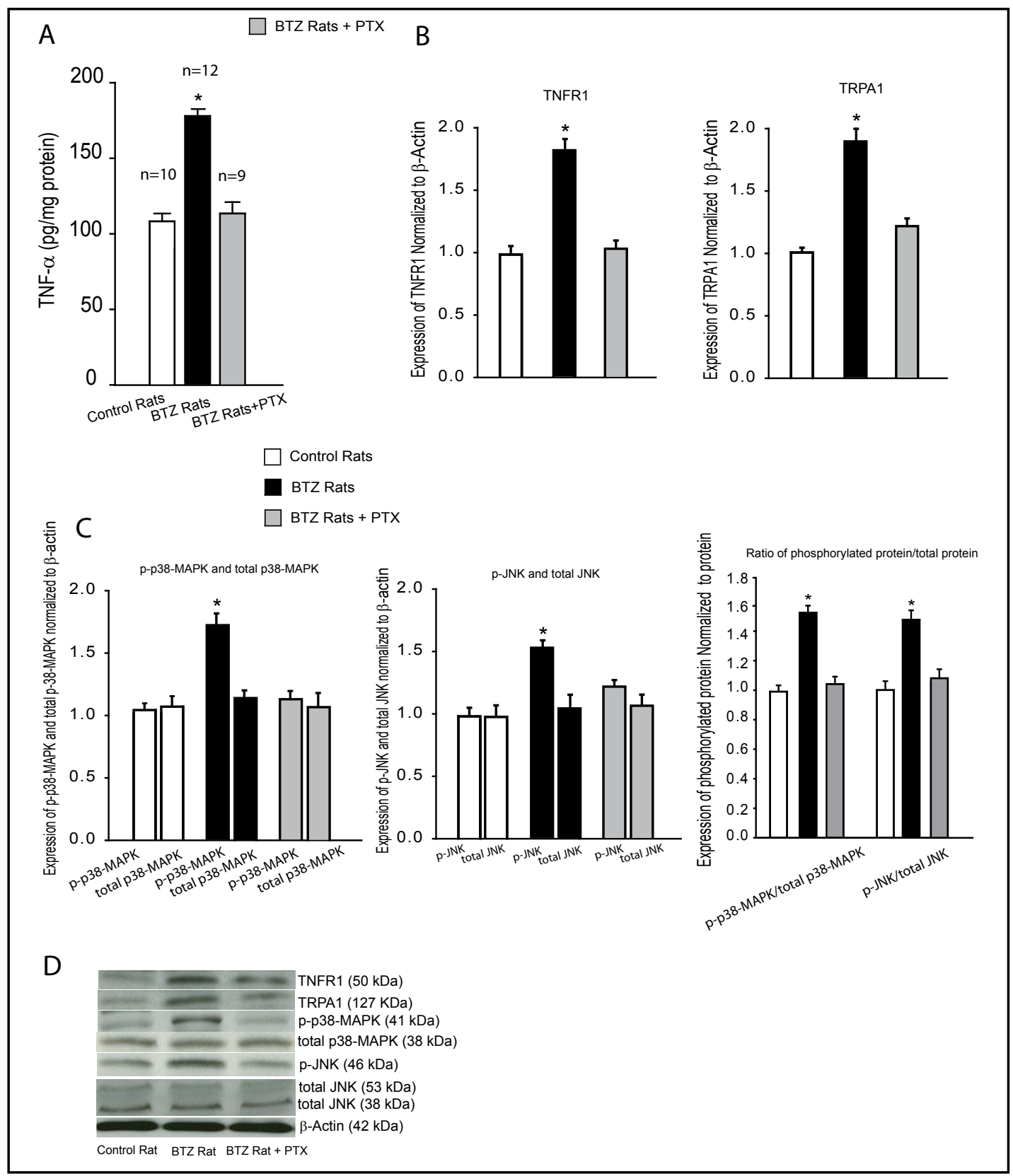

Fig. 4. TNF- $\alpha$ expression and the effects of TNF- $\alpha$ inhibition on signal pathways leading to neuropathic pain. Pentoxifylline (PTX; $40 \mathrm{mg} / \mathrm{kg}$ body weight, i.p. each day for 3 consecutive days) was given to inhibit TNF- $\alpha$. DRG tissues were removed for examination of TNF- $\alpha$ and TRPA1 signal pathways three days after the beginning injection of PTX. (A): The levels of TNF- $\alpha$ were amplified in the DRG tissues of BTZ rats and this increase was inhibited by of PTX. ${ }^{*} \mathrm{P}<0.05$, BTZ rats vs. control rats and BTZ rats that received PTX. The number of rats in each group was shown on the Fig. (B and C): Averaged data show that BTZ increased the protein levels of TNFR1 (a subtype TNF- $\alpha$ receptor) and TRPA1 as well as intracellular signal p-p38-MAPK and p-JNK (phosphorylated form) in the DRG as compared with controls. Furthermore, administration of PTX attenuated increases of these receptor and signal pathways in BTZ animals. Note that the total protein levels of p38-MAPK and JNK were not elevated significantly by BTZ, but the ratio of phosphorylated p38MAPK and JNK and total p38-MAPK and JNK was increased by BTZ. ${ }^{*} \mathrm{P}<0.05$ vs. control rats and BTZ rats with PTX. n=6-8 in each group. Note that averaged total JNK in C was JNK of 53kDa band; and averaged total JNK of $38 \mathrm{kDa}$ was not different among control rats, BTZ rats and BTZ rats with PTX $(\mathrm{P}>0.05$ among three groups). (D): Typical bands demonstrating the results of western blot analysis shown in B\&C. 


\section{Discussion}

One of the most common and distressing symptoms suffered by patients with progression of cancer is pain [21]. Cancer pain mainly arises from a tumor compressing or infiltrating tissue; from nerve and other changes caused by a hormone imbalance or immune response; and/or from treatments and diagnostic procedures [21, 22]. Note that chemotherapy and radiotherapy may produce painful conditions persisting long after treatment has ended [21, $23,24]$. As a result, how to effectively manage cancer pain related to these therapies becomes an important issue for treatment and management of cancer patients in clinics.

BTZ is a compound commonly used to treat multiple myeloma [1-3]. Especially, it has a significant activity against advanced and/or metastatic tumors, but one of the main limiting complications of BTZ is painful neuropathy [3]. Also, a heightened cold sensitivity is another complication observed in cancer patients with BTZ treatment [3].

Prior studies have shown that injection of BTZ can induce neuropathic pain including mechanical hyperalgesia and cold hypersensitivity in rats after initiation of the chemotherapy regimen [16-18]. Of note, the signs of neuropathy start with BTZ therapy. These abnormalities were ablated several days after discontinuation of BTZ [16-18]. Thus, we employed this well established rat model to study the mechanisms of neuropathic pain induced by chemotherapeutic BTZ in the current report. We examined the effects of blocking TRPA1 and TNF- $\alpha$ signal in sensory nerves on mechanical hyperalgesia and cold hypersensitivity in following administration of BTZ.

In prior studies, neuropathic pain was found to be induced in rats by BTZ (0.1-0.5 mg/ $\mathrm{kg}$, i. p., daily over 5 consecutive days) and this persisted for $>7$ days after last injection of BTZ $[16,19]$. On the basis of results of these published work $[16,19]$, we selected the dosage of BTZ and the schedule injecting BTZ in the current report. Consistent with the previous findings [16-18, 25], in this study we observed that BTZ decreased PWT and evoked less time (\%) spent on the cold plate after its injection. Our results further demonstrated that injection of TRPA1 antagonist significantly increased PWT and \% time spent on the cold plate in BTZ rats. It is noted that the effects of HC030031 were also observed in control rats, but the effects of blocking TRPA1 were significantly smaller in BTZ rats as compared with control animals. Likewise, blocking TNF- $\alpha$ had the less effects on PWT and \% time spent on the cold plate in BTZ rats. Consistent with this result, the current study also demonstrated that BTZ increased protein expression of TRPA1 and TNF- $\alpha$ /TNFR1 in the DRG to a greater degree. Interestingly, PTX attenuated TRPA1 in the DRG of control rats and BTZ rats, suggesting the role played by PTX under normal conditions. The same dosage of HC030031 and PTX had the less inhibitory effects on mechanical hyperalgesia and cold hypersensitivity in BTZ rats due to upregulation of TRPA1 and TNFR1. Upregulation of TRPA1 in the DRG of BTZ rats led to mechanical hyperalgesia and cold hypersensitivity. Intracellular signals p38-MAPK and $\mathrm{p}$-JNK are also engaged in the effects of TNF- $\alpha$. Accordingly, our data indicate that BTZ activates TNF- $\alpha$ signal, which subsequently amplifies expression of TRPA1 in the DRG via intracellular p38-MAPK and JNK signals thereby resulting in mechanical hyperalgesia and cold hypersensitivity.

It should be noted that $1.3 \mathrm{mg} / \mathrm{m} 2$ of BTZ (twice weekly for 2 weeks) is a recommended dose used in patients with multiple myeloma [26]. Since average body surface area for adults is $1.7 \mathrm{~m} 2$, each dose of BTZ is $\sim 2.21 \mathrm{mg}$. This is $\sim 0.0295 \mathrm{mg} / \mathrm{kg}$ based on average body weight of $75 \mathrm{~kg}$ for adults. This dosage of BTZ cleared from the body 72-96 hours after its administration [26]. In contrast, in our current study, we injected $0.4 \mathrm{mg} / \mathrm{kg}$ of BTZ daily for 5 consecutive days in each rat. An accumulated amount of BTZ given in a rat was $2 \mathrm{mg} / \mathrm{kg}$ at the end of last BTZ. This dosage is higher than the one used in patients and it is likely to lead to persisting neuropathic pain for several days after last injection of BTZ in the rats as observed in our results and results reported by others [16, 19].

In general, the sensory nerves (neurons) and dorsal horn of the spinal cord primarily conduct signals of pain response. The prior studies have reported that the protein levels of TNF- $\alpha$ are increased in DRG and spinal dorsal horn (within neuronal cells and astrocytes) 
of rats after systemic administration of BTZ-induced neuropathic pain [16, 17]. Consistent with the previous findings $[16,17]$, in the current study we also observed that BTZ increased TNF- $\alpha$ in DRG of rats. Since the similar dosage and approach was used to give BTZ, it is likely that TNF- $\alpha$ was produced in neuronal and glial cells of the DRG in the current study.

A prior human study showed no evidence of demyelination in patient treated with BTZ [27]; whereas a study using mice demonstrated a loss of myelinated fibers of peripheral sensory nerves after BTZ administration [28]. The difference may be due to species and/ or dosages of BTZ used in patients and animals [27, 28]. Nonetheless, this prior animal study further showed that anti-TNF- $\alpha$ alleviated a decrease of density of myelinated fibers. Accordingly, it is well reasoned that inhibition of TNF- $\alpha$ signal was likely to improve demyelination if myelinated fibers of peripheral sensory nerves were decreased in our current study.

TRPA1 has a functional role in regulating pain and neurogenic inflammation resulting from channel activation to a variety of compounds [4-8]. TRPA1 appears in DRG neurons [7] and is engaged in development of mechanical hypersensitivity and painfully cold temperatures $[9,10]$. Additional studies further indicate that TRPA1 mediates mechanical and cold hypersensitivity induced by chemotherapeutic agents $[11,29]$. Our current study suggests that TRPA1 plays a role in regulating BTZ-evoked neuropathic pain. TNF- $\alpha$ is a signal activating TRPA1 because inhibition of TNF- $\alpha$ significantly attenuates BTZ-evoked upregulation of TRPA1 and mechanical hyperalgesia and cold hypersensitivity.

TNF- $\alpha$ is a pro-inflammatory cytokine playing a critical role in the development and maintenance of inflammatory pain [14]. TNF- $\alpha$ induces pain through the release of inflammatory mediators sensitizing ion channels [14]. Our data provided the first evidence that TNF- $\alpha$ has effects on TRPA1 signal pathway by which it mediates mechanical hyperalgesia and cold hypersensitivity induced by BTZ.

TNF- $\alpha$ activates multiple signaling pathways, including the p38-MAPK and JNK pathways [14], which are recognized as important regulators of inflammatory pain. In the prior study using the cultured cells, it was reported that TNF- $\alpha$ amplified TRPA1 via intracellular p38MAPK signal [30]. On the basis of those results, in the current study we designed our in vivo experiments. Indeed, we observed that inhibition of TNF- $\alpha$ by PTX decreased expression of TRPA1, p38-MAPK and JNK in the DRG of BTZ rats and this further attenuated neuropathic pain induced by BTZ. It is reasonably speculated that it would increase TRPA1 expression if TNF- $\alpha$ was applied to cultured DRG cells; and inhibition of p38-MAPK or JNK signal would attenuate the increase of TRPA 1 promoted by TNF- $\alpha$. Nonetheless, our results demonstrated that p38-MAPK and JNK signal pathways are stimulated by BTZ and likely mediate upregulation of TRPA1 in sensory nerves. We also demonstrated, for the first time, a novel mechanism by which TNF- $\alpha$ contributes to enhanced TRPA1 expression likely via p38-MAPK and JNK signal, which are involved in mechanical hyperalgesia and cold hypersensitivity induced by BTZ.

In addition to TNF- $\alpha$, other pro-inflammatory cytokines including IL- 6 and IL- $1 \beta$ have been reported to be involved in BTZ-induced neuropathic pain $[16,17]$. For example, IL- 6 was elevated in the circulation of patients with BTZ treatment and its levels were correlated with the appearance of BTZ-induced neuropathic pain [31]. IL-1 $\beta$ and IL-6 were elevated in the DRG of rats with injection of BTZ [28]. BTZ treatment induced upregulation of the mRNA and protein levels of IL- $1 \beta$ in the astrocytes of the spinal dorsal horn of rats and this also increased the phosphorylation of JNK 17. Inhibition of IL-1 $\beta$ and TNF- $\alpha$ alleviated JNK activation and lessened mechanical allodynia induced by BTZ $[16,17]$.

JNK is activated by both TNF- $\alpha$ and IL-1 $\beta$ in cultured DRG cells [32, 33]. ERK and p38MAPK are also downstream molecules of TNF- $\alpha$ - and IL-1 $\beta$-mediated signaling. A prior study reported that intrathecal injection of TNF- $\alpha$ or IL-1 $\beta$ increased the phosphorylation of p38MAPK, but not that of ERK and JNK in the spinal cord in mice [34]. Another study suggests that inhibition of TNF- $\alpha$ or IL-1 $\beta$ significantly suppressed BTZ-induced JNK activation [17]. These discrepancies may result from the different animal models, experimental preparations, doses of blocking agents and/or drugs used in the studies and behavior testing times etc. 
Nonetheless, in the current study, blocking TNF- $\alpha$ using PTX attenuated the upregulation of p38-MAPK and JNK in the DRG induced by BTZ and thereby alleviated mechanical and cold hypersensitivity.

Calcium is a key regulator of major cellular processes. Its cytosolic concentration is determined mainly by extracellular calcium influx, release of calcium from internal stores, and mitochondrial uptake. The levels of calcium and its channel activity contribute to pathophysiological process of pain [35]. It has been reported that mitochondrial-mediated dysregulation of calcium homeostasis or dysregulation of neurotrophins are involved in the mechanism of BTZ-induced neuropathy $[36,37]$. A prior study suggests that calcium influx is a part of process for upregulation of TRPA1 expression by TNF- $\alpha$ [38]. Also, as TRPA1 receptor in sensory neurons is activated calcium influx occurs in involvement of pain response [35]. In the current study, we observed that blocking TNF- $\alpha$ attenuated TRPA1 expression and decreased mechanical and cold sensitivity. Thus, we speculated that the effects of blocking TNF- $\alpha$ would improve dysregulation of calcium homeostasis in attenuating neuropathic pain. In addition, increases in neurotrophins (such as nerve growth factor) can amplify pain response and upregulation of TRPA1 expression and increases of TRPA1 activity are partly involved in the effects of neurotrophins $[39,40]$. Thus, enhancement of TRPA1 observed in this study is likely linked to dysregulation of neurotrophins in BTZ-induced neuropathy [36].

It needs to be acknowledged that TNF- $\alpha$ is a player in regulating the biological process of tumor development. Prior studies suggest a linkage between TNF- $\alpha$ and hematologic and non-hematologic malignancy [41]. While some studies showed that a high level of TNF- $\alpha$ suppresses the tumor angiogenesis in malign tissues, the other studies showed that TNF- $\alpha$ may act as an endogenous tumor growth factor [41]. BTZ is used for treatment of multiple myeloma. Results from our present study and other reports showed that TNF- $\alpha$ signal is amplified by BTZ intervention $[16,17]$. This might be a mechanism to be beneficial to treatment of multiple myeloma if elevated TNF- $\alpha$ plays an inhibitory role in the tumor angiogenesis in malign tissues. On the other hands, TNF- $\alpha$ signal is engaged in neuropathic pain, a side effect caused by BTZ as shown in many studies $[26,36]$. Thus, a better understanding of TNF- $\alpha$ signal pathway during BTZ intervention seems more profound.

Finally, the effects of HC030031 and PTX on locomotor activities need to be addressed. A prior study suggested that HC030031 attenuates mechanical hyperalgesia induced by cyclophosphamide without interfere with locomotor activity in rats [42]. Another study also indicated that PTX fails to alter a decrease in locomotor activities induced by HIV-1 glycoprotein 120 in rats [43]. Thus, in the current study it is unlikely that the effects of HC030031 and PTX on mechanical and cold hypersensitivity were affected by locomotor activities per se.

\section{Conclusion}

In conclusion, the protein expression levels of TRPA1 receptors in peripheral sensory nerves are upregulated by injection of BTZ; inhibition of TRPA1 and TNF- $\alpha$ signal antagonizes mechanical hyperalgesia and cold hypersensitivity during BTZ intervention; and TRPA1 pathways play a role in TNF- $\alpha$ engagement of BTZ-induced neuropathic pain via intracellular p38-MAPK and JNK signal. Results of this study provide a base for the mechanisms responsible for BTZ-induced neuropathic pain. In particular, targeting one or more of these signaling molecules involved in activation of TRPA1 and TNF- $\alpha$ evoked by BTZ may present new opportunities for treatment and management of neuropathic pain in patients with multiple myeloma during BTZ chemotherapeutics. 


\section{Cellular Physiology Cell Physiol Biochem 2018;51:2098-2110

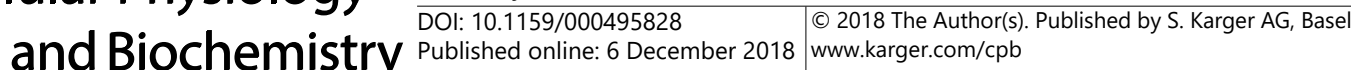 \\ Li et al.: Neuropathic Pain During Chemotherapeutic Intervention}

\section{Acknowledgements}

This study was supported by the Natural Science Foundation of Hubei Province (No. 2016CFB545).

\section{Disclosure Statement}

The authors declare no competing interests.

\section{References}

1 Adams J, Kauffman M: Development of the proteasome inhibitor Velcade (Bortezomib). Cancer Invest 2004;22:304-311.

2 Voorhees PM, Dees EC, O'Neil B, Orlowski RZ: The proteasome as a target for cancer therapy. Clin Cancer Res 2003;9:6316-6325.

-3 Curran MP, McKeage K: Bortezomib: a review of its use in patients with multiple myeloma. Drugs 2009;69:859-888.

4 Andersson DA, Gentry C, Moss S, Bevan S: Transient receptor potential A1 is a sensory receptor for multiple products of oxidative stress. J Neurosci 2008;28:2485-2494.

-5 Bandell M, Story GM, Hwang SW, Viswanath V, Eid SR, Petrus MJ, Earley TJ, Patapoutian A: Noxious cold ion channel TRPA1 is activated by pungent compounds and bradykinin. Neuron 2004;41:849-857.

6 Bautista DM, Movahed P, Hinman A, Axelsson HE, Sterner O, Hogestatt ED, Julius D, Jordt SE, Zygmunt PM: Pungent products from garlic activate the sensory ion channel TRPA1. Proc Natl Acad Sci U S A 2005;102:12248-12252.

7 Jordt SE, Bautista DM, Chuang HH, McKemy DD, Zygmunt PM, Hogestatt ED, Meng ID, Julius D: Mustard oils and cannabinoids excite sensory nerve fibres through the TRP channel ANKTM1. Nature 2004;427:260265.

-8 Sawada Y, Hosokawa H, Matsumura K, Kobayashi S: Activation of transient receptor potential ankyrin 1 by hydrogen peroxide. Eur J Neurosci 2008;27:1131-1142.

\$9 Kwan KY, Allchorne AJ, Vollrath MA, Christensen AP, Zhang DS, Woolf CJ, Corey DP: TRPA1 contributes to cold, mechanical, and chemical nociception but is not essential for hair-cell transduction. Neuron 2006;50:277-289.

10 Story GM, Peier AM, Reeve AJ, Eid SR, Mosbacher J, Hricik TR, Earley TJ, Hergarden AC, Andersson DA, Hwang SW, McIntyre P, Jegla T, Bevan S, Patapoutian A: ANKTM1, a TRP-like channel expressed in nociceptive neurons, is activated by cold temperatures. Cell 2003;112:819-829.

-11 Zhao M, Isami K, Nakamura S, Shirakawa H, Nakagawa T, Kaneko S: Acute cold hypersensitivity characteristically induced by oxaliplatin is caused by the enhanced responsiveness of TRPA1 in mice. Mol Pain 2012;8:55.

12 Clark AK, Old EA, Malcangio M: Neuropathic pain and cytokines: current perspectives. J Pain Res 2013;6:803-814.

13 Mika J, Zychowska M, Popiolek-Barczyk K, Rojewska E, Przewlocka B: Importance of glial activation in neuropathic pain. Eur J Pharmacol 2013;716:106-119.

14 Miller RJ, Jung H, Bhangoo S, White FA: Cytokine and chemokine regulation of sensory neuron function; in (Canning BJ, and Spina DV, eds) Handbook of Experimental Pharmacology. Berlin Heidelberg, Springer, 2009, vol. pp. 417-449.

15 Carozzi VA, Canta A, Chiorazzi A: Chemotherapy-induced peripheral neuropathy: What do we know about mechanisms? Neurosci Lett 2015;596:90-107.

16 Zhang J, Su YM, Li D, Cui Y, Huang ZZ, Wei JY, Xue Z, Pang RP, Liu XG, Xin WJ: TNF-alpha-mediated JNK activation in the dorsal root ganglion neurons contributes to Bortezomib-induced peripheral neuropathy. Brain Behav Immun 2014;38:185-191. 


\section{Cellular Physiology Cell Physiol Biochem 2018;51:2098-2110 \begin{tabular}{l|l|l|l|l}
\hline DOI: 10.1159/000495828 & $\begin{array}{l}\text { C } 2018 \text { The Author(s). Published by S. Karger AG, Basel } \\
\text { www.karger.com/cpb }\end{array}$
\end{tabular}

17 Li ZY, Zhang YP, Zhang J, Zhang SB, Li D, Huang ZZ, Xin WJ: The possible involvement of JNK activation in the spinal dorsal horn in bortezomib-induced allodynia: the role of TNF-alpha and IL-1beta. J Anesth 2016;30:55-63.

18 Chiorazzi A, Canta A, Meregalli C, Carozzi V, Sala B, Oggioni N, Monbaliu J, H VDV, Cavaletti G: Antibody against tumor necrosis factor-alpha reduces bortezomib-induced allodynia in a rat model. Anticancer Res 2013;33:5453-5459.

19 Zheng H, Xiao WH, Bennett GJ: Mitotoxicity and bortezomib-induced chronic painful peripheral neuropathy. Exp Neurol 2012;238:225-234.

-20 Chaplan SR, Bach FW, Pogrel JW, Chung JM, Yaksh TL: Quantitative assessment of tactile allodynia in the rat paw. J Neurosci Methods 1994;53:55-63.

21 Hanna, Magdi, Zylicz, Zbigniew: Cancer Pain. Springer 2013.

22 Pasetto LM, D’Andrea MR, Rossi E, Monfardini S: Oxaliplatin-related neurotoxicity: How and why? Critical Reviews in Oncology/Hematology 2006;59:159-168.

23 Hoskin PJ: Radiotherapy; in Clinical pain management: Cancer Pain. London, Hodder Arnold, 2008, vol. p.^pp. 251-255.

24 Portenoy RK: Treatment of cancer pain. Lancet 2011;377:2236-2247.

25 Cavaletti G, Gilardini A, Canta A, Rigamonti L, Rodriguez-Menendez V, Ceresa C, Marmiroli P, Bossi M, Oggioni N, D’Incalci M, De Coster R: Bortezomib-induced peripheral neurotoxicity: a neurophysiological and pathological study in the rat. Exp Neurol 2007;204:317-325.

26 Schwartz R, Davidson T: Pharmacology, pharmacokinetics, and practical applications of bortezomib. Oncology (Williston Park) 2004;18:14-21.

27 Dai X, Sun X, Ni H, Zhu X: Guillain Barre Syndrome in a multiple myeloma patient after the first course of bortezomib therapy: A case report. Oncol Lett 2015;10:3064-3066.

-28 Ale A, Bruna J, Morell M, van de Velde H, Monbaliu J, Navarro X, Udina E: Treatment with anti-TNF alpha protects against the neuropathy induced by the proteasome inhibitor bortezomib in a mouse model. Exp Neurol 2014;253:165-173.

29 Nassini R, Fusi C, Materazzi S, Coppi E, Tuccinardi T, Marone IM, De Logu F, Preti D, Tonello R, Chiarugi A, Patacchini R, Geppetti P, Benemei S: The TRPA1 channel mediates the analgesic action of dipyrone and pyrazolone derivatives. Br J Pharmacol 2015;172:3397-3411.

-30 El Karim I, McCrudden MT, Linden GJ, Abdullah H, Curtis TM, McGahon M, About I, Irwin C, Lundy FT: TNFalpha-induced p38MAPK activation regulates TRPA1 and TRPV4 activity in odontoblast-like cells. Am J Pathol 2015;185:2994-3002.

31 Mangiacavalli S, Corso A, De Amici M, Varettoni M, Alfonsi E, Lozza A, Lazzarino M: Emergent T-helper 2 profile with high interleukin-6 levels correlates with the appearance of bortezomib-induced neuropathic pain. Br J Haematol 2010;149:916-918.

32 Hou L, Li W, Wang X: Mechanism of interleukin-1 beta-induced calcitonin gene-related peptide production from dorsal root ganglion neurons of neonatal rats. J Neurosci Res 2003;73:188-197.

-33 Pollock J, McFarlane SM, Connell MC, Zehavi U, Vandenabeele P, MacEwan DJ, Scott RH: TNF-alpha receptors simultaneously activate $\mathrm{Ca} 2+$ mobilisation and stress kinases in cultured sensory neurones. Neuropharmacology 2002;42:93-106.

34 Meotti FC, Posser T, Missau FC, Pizzolatti MG, Leal RB, Santos AR: Involvement of p38MAPK on the antinociceptive action of myricitrin in mice. Biochem Pharmacol 2007;74:924-931.

-35 Bourinet E, Altier C, Hildebrand ME, Trang T, Salter MW, Zamponi GW: Calcium-permeable ion channels in pain signaling. Physiol Rev 2014;94:81-140.

-36 Argyriou AA, Iconomou G, Kalofonos HP: Bortezomib-induced peripheral neuropathy in multiple myeloma: a comprehensive review of the literature. Blood 2008;112:1593-1599.

-37 Siau C, Bennett GJ: Dysregulation of cellular calcium homeostasis in chemotherapy-evoked painful peripheral neuropathy. Anesth Analg 2006;102:1485-1490.

38 Meng J, Wang J, Steinhoff M, Dolly JO: TNFalpha induces co-trafficking of TRPV1/TRPA1 in VAMP1containing vesicles to the plasmalemma via Munc18-1/syntaxin1/SNAP-25 mediated fusion. Sci Rep 2016;6:21226.

39 Devesa I, Ferrer-Montiel A: Neurotrophins, endocannabinoids and thermo-transient receptor potential: a 


\section{Cellular Physiology Cell Physiol Biochem 2018:51:2098-2110 \begin{tabular}{ll|l} 
DOI: 10.1159/000495828 & 2018 The Author(s). Published by S. Karger AG, Basel \\
and Biochemistry & Published online: 6 December 2018 ww.com/cpb \\
\cline { 2 - 3 }
\end{tabular}}

threesome in pain signalling. Eur J Neurosci 2014;39:353-362.

40 Diogenes A, Akopian AN, Hargreaves KM: NGF up-regulates TRPA1: implications for orofacial pain. J Dent Res 2007;86:550-555.

41 Wajant H, Pfizenmaier K, Scheurich P: Tumor necrosis factor signaling. Cell Death Differ 2003;10:45-65.

-42 Meotti FC, Forner S, Lima-Garcia JF, Viana AF, Calixto JB: Antagonism of the transient receptor potential ankyrin 1 (TRPA1) attenuates hyperalgesia and urinary bladder overactivity in cyclophosphamide-induced haemorrhagic cystitis. Chem Biol Interact 2013;203:440-447.

43 Barak 0, Goshen I, Ben-Hur T, Weidenfeld J, Taylor AN, Yirmiya R: Involvement of brain cytokines in the neurobehavioral disturbances induced by HIV-1 glycoprotein120. Brain Res 2002;933:98-108. 